Case Report

\title{
Etiological profile and outcome of hypertransaminemia in children
}

\author{
Sankar J. ${ }^{1}$, Ramesh V. ${ }^{2}$, Nagamalleswari ${ }^{3}$ \\ ${ }^{1}$ Dr. Janani Sankar, Senior Consultant, ${ }^{2}$ Dr. Venkateswari Ramesh, Junior Consultant, ${ }^{3}$ Nagamalleswari, Registrar, all \\ authors are affiliated with Department of Pediatrics, Kanchi Kamakoti Childs Trust Hospital, 12a, Nageswara Road, \\ Nungambakkam, Chennai, Tamilnadu, India.
}

Corresponding Author: Dr. Janani Sankar, Senior Consultant, Department of Pediatrics, Kanchi Kamakoti Childs Trust Hospital, 12a, Nageswara Road, Nungambakkam, Chennai, Tamilnadu, India. Email: janani.sankar@yahoo.com

\begin{abstract}
Objectives: To study the causes of elevated transaminases in children and assess their outcome. Material and Methods: Retrospective study conducted at Kanchi Kamakoti CHILDS trust hospital, Chennai for 6 months. Medical records of 305 children with hypertransaminemia were analyzed for identifying etiology, their utility in diagnosis as well as predicting outcome. Results: The commonest etiology of elevated transaminases were infections. Highest levels of elevation were recorded in drug induced liver injury and shock. Massive elevation of transaminases along with deranged prothrombin time and albumin was associated with greater mortality and morbidity. Conclusions: Serum transaminases may be adapted as an improtant investigation, not only to detect liver dysfunction but also to diagnose and prognosticate several disaeses which are non-hepatic in origin.
\end{abstract}

Keywords: Transaminases, Paracetamol toxicity, Paracetamol toxicity, Hypertransaminemia

\section{Introduction}

Aminotransferases are normally present in circulation at low levels. They are intracellular enzymes produced principally by hepatocytes, and their increase in serum is therefore indicative of liver cell injury. However, aspartate aminotransferase (AST) is also found in cardiac and skeletal muscles, the kidneys, brain, pancreas, and lungs, and in erythrocytes, in decreasing order of concentration. Additionally, alanine aminotransferase (ALT) is present in skeletal muscle and kidneys, but at low concentrations, and its increase in the circulation is more specific for liver damage than AST. Aminotransferase serum levels depend not only on the tissue of origin, but also on the enzyme half-life, which is longer for ALT than AST.

Thus, in diseases such as muscular dystrophy, patients can have AST and ALT serum values that are elevated to the same degree, instead of the expected prevalent elevation of AST. In clinical practice, normal parameter values are within 2 standard deviations of the mean value obtained in healthy individuals. Serum transaminases namely Aspartate aminotransferase (AST) and alanine transferase (ALT), are the most frequently utilised, noninvasive and sensitive indicators of liver

Manuscript received: $10^{\text {th }}$ September 2018

Reviewed: $20^{\text {th }}$ September 2018

Author Corrected: $26^{\text {th }}$ September 2018

Accepted for Publication: $30^{\text {th }}$ September 2018 injury. ALT is primarily localized to the liver but the AST is present in a wide variety of tissues like the heart, skeletal muscle, kidney, brain and liver. Apart from primary liver disease and muscle disorders they can be elevated in variable conditions like viral or bacterial infection, drug toxicity, systemic illnesses and inflammation.

The variation in pattern and extent of elevation among different diseases coupled with their easy availability and sensitivity, makes them an important tool in diagnosing certain diseases as well as assessing their severity $[1,2]$. There is a definite underutilization of transaminase testing and application of its elevation as a clue to the diagnosis of underlying illness.

The present study aims to stress the usefulness of testing for transaminase levels, identify the most common causes of hypertransaminemia in children, their utility in diagnosis and as predictors of outcome.

\section{Aims and Objectives}

To study the etiological profile of children with elevated serum transaminases.

To assess the outcome of these children and identify the predictors of outcome. 


\section{Material and Methods}

Place of study: Kanchi Kamakoti CHILDS Trust Hospital, Chennai.

Type of study: Prospective Descriptive study

Duration of study: Oct 2016 - Mar 2017

Inclusion Criteria: This comprised of children from newborn period to eighteen years of age, attending the outpatient departments as well as those admitted in wards and intensive care units for various reasons, including those not related to liver disorders.

\section{Case Report}

Sampling Methods: The case records of these children were retrospectively analysed for etiology, demographic data, clinical presentation, other laboratory parameters and final outcome.

An elevation of 3 times the upper limit of normal values for serum transaminases was taken as an arbitrary cut off and 305 children with hyper transaminemia were identified from the biochemistry lab register.

An attempt was made to identify the overall predictors for need for intensive care and mortality, especially with regards to dengue fever and paracetamol poisoning

\section{Results}

A total of 305 children with elevated transaminases were identified during the study period. This study group comprised of $172(56.4 \%)$ males and $133(43.6 \%)$ female children, including $29(9.5 \%)$ neonates. 32 (10.5\%) children were born to consanguineous parents. Majority of the children underwent investigations as a part of work up for fever $(n=184,60.3 \%)$ and associated vomiting $(n=63,20.6 \%)$, abdominal pain $(n=43,14.1 \%)$ and lethargy $(n=35,11.5 \%)$. Only $49(16.1 \%)$ children were being evaluated for jaundice.

Most common cause of elevated transaminases were infections ( $\mathrm{n}=142,46.2 \%)$ out of which majority were due to dengue infection $(n=52,17.5 \%)$, viral fever $(n=39,12.8 \%)$, enteric fever $(n=15,4.9 \%)$ and sepsis $(n=13,4.3 \%)$. Liver and biliary disorders per se were identified in 31(10.2\%) children, commonest being metabolic disease $(n=16,51.6 \%)$ and surgical conditions $(n=11,35.5 \%) .13$ children had drug or toxin related liver injury $(n=12,3.9 \%)$, with paracetamol $(n=$ 9, 69.2\%) identified as the leading agent. Elevation of liver enzymes was also found as a part of systemic inflammatory disorders like HLH $(n=8,2.6 \%)$ and severe systemic illnesses culminating in $\operatorname{MODS}(n=7,2.3 \%)$.

Highest value of transaminases $(>10,000 \mathrm{u} / 1)$ was found in paracetamol toxicity and circulatory shock. AST: ALT ratios were found to be less than 1 in $77.8 \%$ of children with viral hepatitis and $70 \%$ of those with paracetamol induced liver injury.

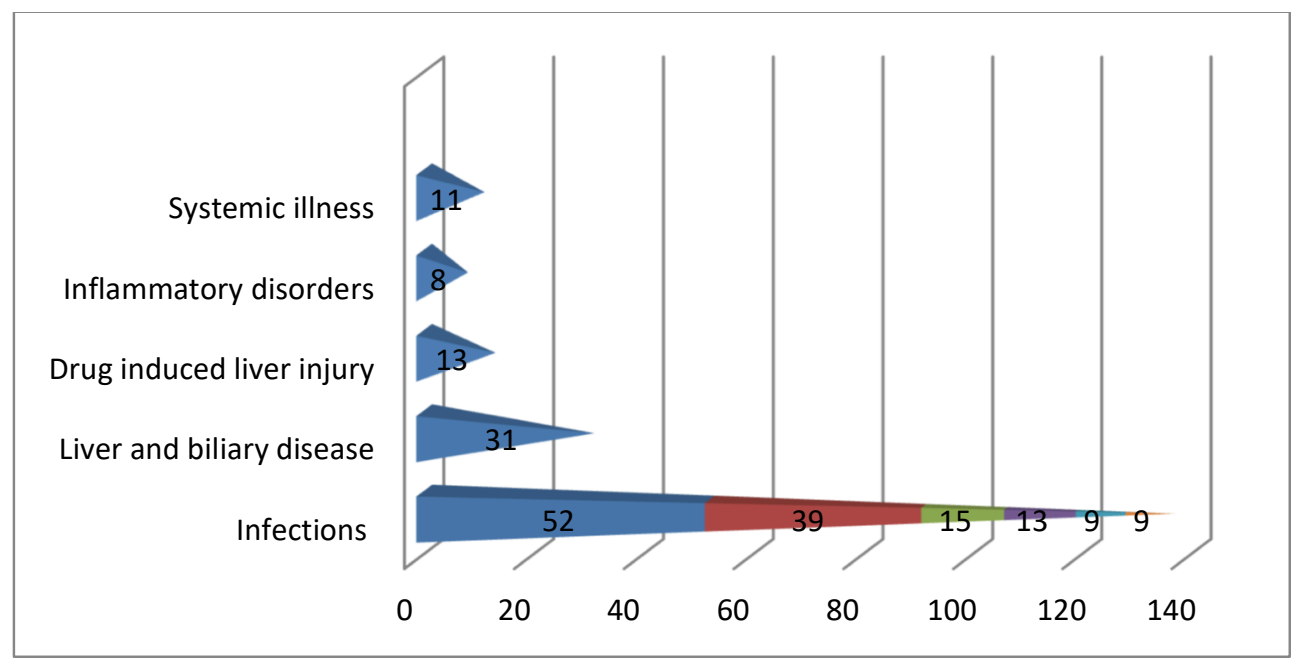

Fig-1: Etiological profile of hypertransaminemia

In all the 10 cases of paracetamol over dosage, children presented with a co -existing febrile illness associated with disproportionate lethargy and the diagnosis was made on a retrospective basis only after reviewing the liver function abnormalities. 7 (70\%) out of these children required intensive care and all of them survived. All these 7 (100\%) children had an elevated prothrombin time / INR and 3(42.8\%) had associated renal injury and metabolic acidosis requiring hemodilaysis. 
Case Report

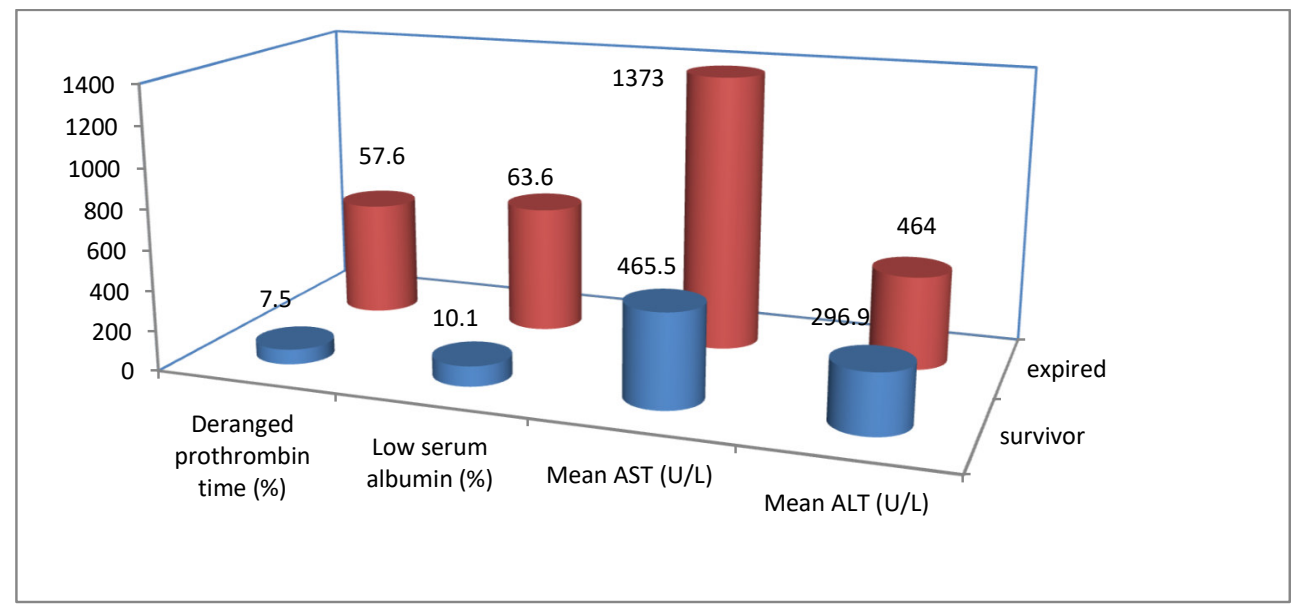

Fig-2: Predictors of mortality

Their transaminases were massively elevated to the tune of $5266.8 \mathrm{u} / 1$ (AST) and $2799 \mathrm{u} / 1$ (ALT) as compared to their non ICU counterparts $(1318 \mathrm{u} / 1$ and $519 \mathrm{u} / \mathrm{l})$ respectively $8(15.2 \%)$ out of the 52 children with dengue fever were admitted in ICU care. Low serum albumin $(n=5,62.5 \%)$ and third spacing with respiratory distress $(n=4,50 \%)$ were the most common associated findings.

$33(10.1 \%)$ children expired during the course of study period. Mortality was highest in infections associated with sepsis $(\mathrm{n}=13,39.4 \%)$, neonates being the most affected $(\mathrm{n}=8,61.5 \%)$. The mean values of AST and ALT in children who succumbed to death due to various reasons was $1373 \mathrm{U} / \mathrm{L}$ and $464 \mathrm{u} / 1$ respectively as compared to $465.5 \mathrm{u} / 1$ and $296.9 \mathrm{u} / 1$ in children who improved. An elevated prothrombin time and INR was found in 57.6\% and serum albumin values were low in $63.6 \%$ of these children as compared to $7.5 \%$ and $10.1 \%$ respectively in those who survived.

\section{Discussion}

Individual patients can have baseline fluctuations in serum aminotransferase levels. The sensitivity and specificity of the serum aminotransferases, used to discriminate those with and without liver disease, depend on the cutoff values chosen to define an abnormal test [3]. There is, however, a poor correlation between degree of liver damage and the levels of transaminases. The absolute elevation of aminotransferases is thus of no prognostic significance in acute hepatocellular disorder.

The ratio of AST to ALT at times gives a clue to the likely cause. In many acute and chronic liver disease or steatosis the ratio is less than or equal to 1. An AST: ALT ratio greater than 2 is suggestive of alcoholic hepatitis. Wilson's disease related hepatitis could cause the AST: ALT ratio to exceed[4]. Within the population studied, 87 percent of patients with an AST: ALT ratio of 1.3 or less had NASH $(87 \%$ sensitivity, $84 \%$ specificity). As the severity of NASH increased, so did the AST: ALT ratio [5].

The transaminases alanine aminotransferase (ALT) and aspartate aminotransferase (AST) are markers of hepatocellular injury but are highly concentrated in muscle cells. Consequently, muscular dystrophies such as Duchenne muscular dystrophy, lead to hyper transaminasemia. Elevation in ALT and AST is most striking during the early stages of disease, before onset of or when only subtle signs of muscle disease are present. Thus, the incidental finding of elevated ALT/AST may be the presenting sign of muscle disease in many children and provides an opportunity for early diagnosis. In a study done by Wright $\mathrm{M}$ et al it was found that there was a delay in diagnosis of DMD as these children were extensivelyworked up for liver disease This results in delayed diagnosis and initiation of treatment and increased expense and may lead to unnecessary invasive procedures [6].

Genetic disease accounted for $12 \%$ of cases of isolated hyper transaminasemia observed in a tertiary pediatric department. A high level of suspicion is desirable for an early diagnosis of these disorders, which may present with isolated hyper transaminasemia and absence of typical clinical signs [7].

The most common causes of elevated transaminases in our study were infections, metabolic and surgical liver disease, drug related toxicity which is comparable to earlier Indian studies [3]. 


\section{Case Report}

Massive elevation of transaminases was recorded in drug induced hepatitis and ischemic liver injury due to shock, as mentioned in the existing literature [2].

AST: ALT ratio was found to be less than 1 in majority of children with viral hepatitis and paracetamol toxicity. Though AST: ALT ratio has been used as a marker for cirrhosis and alcoholic disease in adults, pediatric experience is limited.

Higher degree of elevation of transaminases along with deranged prothrombin time and low serum albumin were associated with greater need for intensive care and mortality, especially in paracetamol over dosage and dengue infections. There is lack of studies regarding role of transaminases in predicting mortality and morbidity. Persistent hypertransaminasemia can be due to metabolic, genetic, gastrointestinal, and extrahepatic causes that should be considered in clinical practice. Importantly, information derived from the combination of the patient's history, physical examination, and basic laboratory data are necessary to reach a timely and correct diagnosis

\section{Conclusions}

Majority of children with elevated liver enzymes had infections rather than liver disease. Significant elevation of liver enzymes, AST: ALT ratio less than 1 or disproportionate lethargy in a child with routine febrile illness may point towards a co- existing paracetamol over dosage.

The intensity of elevation in liver enzymes and associated factors like elevated prothrombin time may be used to assess severity of disease, need for intensive care andas a predictor for mortality especially in conditions like dengue and drug induced liver injury.

The present study has certain limitations- it is a retrospective study and the follow up duration was short. A longer follow up period is required to identify the actual morbidity and mortality.

\section{What is already Known?}

Transaminase elevation is usually seen in Liver diseases and is used to prognosticate Liver cell dysfunction.

\section{What this study adds?}

Transaminases are elevated in many systemic infections and the variation in pattern and extent of elevation among different diseases coupled with their easy availability and sensitivity, makes them an important tool in diagnosing certain diseases as well as assessing their severity.

Funding: Nil, Conflict of interest: None initiated, Perission from IRB: Yes

\section{References}

1. Ki-Soo Kang. Review article. Abnormality in liver function test. Pediatr Gastroenterol HepatolNutr 2013 December 16(4):225-232

2. Thapa BR, Walia A. Liver function tests and their interpretation. Indian J Pediatr. 2007 Jul;74(7):663-71.

3. Ahmar S, Nimain CM. Etiological spectrum of children presenting with raised liver transaminases and their outcome in a tertiary care pediatric facility at Navi Mumbai, India. Clinical Gastroenterology and hepatology. Elsevier Inc. 2015.

4. Davern TJ, Scharschmidt BF. Biochemical liver tests. In: Feidman M, Friedman LS, Sleissenger MH, eds. Sleisenger and Fordtrans Gastrointestinal and liver disease: pathophysiology, diagnosis, management.7th ed. Philadelphia: Saunders; 2002:1227-38.

5. Sorbi D, Boynton J, Lindor KD. The ratio of aspartate aminotransferase to alanine aminotransferase: potential value in differentiating nonalcoholic steatohepatitis from alcoholic liver disease. Am J Gastroenterol. 1999 Apr;94(4):1018-22.doi:10.1111/j. 15720241.1999.01006.x

6. Iorio R, Sepe A, Giannattasio A, et al. Hypertransaminasemia in childhood as a marker of genetic liver disorders. J Gastroenterol. 2005 Aug;40 (8):820-6. DOI:10.1007/s00535-005-1635-7

7. Wright MA, Yang ML, Parsons JA, et al. Consider muscle disease in children with elevated transaminase. $\mathrm{J}$ Am Board Fam Med. 2012 Jul-Aug;25(4):536-40. doi: 10.3122/jabfm.2012.04.110183.

\section{How to cite this article?}

Sankar J, Ramesh V, Nagamalleswari. Etiological profile and outcome of hypertransaminemia in children. Int J Pediatr Res.2018;5(9):451-454. doi:10.17511/ijpr.2018.i09.04. 